\title{
An Iterative Detection Technique for DS-CDMA Signals with Strong Nonlinear Distortion Effects
}

\author{
Rui Dinis and Paulo Silva \\ ISR-IST, Tech. Univ. of Lisbon, Av. Rovisco Pais, 1049-001 Lisboa, Portugal, \\ Phone: +351 218418298; Fax: +351 218418291; email: rdinis@ist.utl.pt
}

\begin{abstract}
Whenever a DS-CDMA signal (Direct Sequence Code Division Multiple Access) is the sum of several components associated to different spreading codes (e.g., the DS-CDMA signal to be transmitted by the BS (Base Station) in the downlink, or any multicode DSCDMA signal), it has high envelope fluctuations, and a high PMEPR (Peak-to-Mean Envelope Power Ratio) setting strong linearity requirements for the power amplifiers. For this reason, it is desirable to reduce the envelope fluctuations of the transmitted signals.
\end{abstract}

A promising class of nonlinear signal processing schemes, combining a nonlinear operation in the timedomain with a linear, frequency-domain filtering operation, was recently proposed for reducing the envelope fluctuations (and, inherently, the PMEPR) of DS-CDMA signals, while maintaining the spectral occupation of the corresponding conventional DS-CDMA signals. However, the performance degradation due to the nonlinear distortion effects on the transmitted signals can be relatively high, especially when a low PMEPR is intended. This can be especially serious if different powers are assigned to different spreading codes.

To avoid significant performance degradation in these situations, we consider improved receiver where there is an iterative estimation and cancelation of nonlinear distortion effects.

Our performance results show that the proposed receiver allows significant performance improvements after just a few iterations, even when we have strong nonlinear distortion effects. ${ }^{1}$

//Keywords: DS-CDMA, envelope fluctuations, iterative detection, signal processing.

\section{Introduction}

The DS-CDMA (Direct Sequence Code Division Multiple Access) schemes have been selected for several wireless systems, namely due to their good capacities and performances in time-dispersive channels [1], [2]. These techniques are good cadidates for broadband wireless systems, especially when combined with CP-assisted (Cyclic Prefix) block transmission

\footnotetext{
${ }^{1}$ This work was partially supported by the FCT project POSI/CPS/46701/2002 - MC-CDMA and the IST C-MOBILE project.
}

techniques and FDE schemes (Frequency-Domain Equalization) [3], [4].

However, the envelope fluctuations and the PMEPR (Peakto-Mean Envelope Power Ratio) of DS-CDMA signals can be very high when we combine a large number of signals with different spreading codes, namely at the downlink transmission and/or for multicode CDMA schemes [5], since the high PMEPR of the DS-CDMA signals leads to amplification difficulties. For this reason, several techniques were proposed for designing low-PMEPR DS-CDMA signals, e.g., by adding unused codes [6] or through suitable signal processing techniques (see [7] and references therein).

A promising class of nonlinear signal processing schemes for reducing the envelope fluctuations (and, inherently, the PMEPR) of DS-CDMA signals was proposed in [8]. These schemes combine a nonlinear operation in the time-domain, followed by a linear, frequency-domain filtering operation to reduce significantly the PMEPR of the transmitted signals, while maintaining the spectral occupation of the corresponding conventional DS-CDMA signals. However, the performance degradation due to the nonlinear distortion effects on the transmitted signals can be relatively high, especially when a low PMEPR is intended and/or different powers are assigned to different spreading codes.

It was shown in [9], [10] that we can improve significantly the performance of nonlinearly-distorted OFDM schemes (Orthogonal Frequency Division Multiplexing) by employing an enhanced receiver structure with iterative cancelation of nonlinear distortion effects.

In this paper we consider the techniques of [8] for reducing the PMEPR of DS-CDMA signals and we propose an improved receiver where the nonlinear distortion effects are estimated and canceled in an iterative way, so as to improve the performances when a low-PMEPR DS-CDMA transmission is intended.

The paper is organized as follows: In sec. II, we describe the transmitter structure. Sec. III, describes the receiver structure proposed in this paper. A set of performance results is presented in sec. IV and sec. V is concerned with the conclusions of this paper.

\section{Transmitter Structure}

In this paper we consider the downlink transmission in DS-CDMA systems employing CP-assisted block transmission 
techniques, combined with FDE schemes (Our approach could easily be extended to conventional DS-CDMA schemes; the extension to multicode schemes is also straightforward.). The BS (Base Station) transmits simultaneously data blocks for $K_{U}$ users. For the sake of simplicity, it is assumed that all users have the same spreading factor (the generalization to other cases is straightforward). The block of chips transmitted by the BS is $\left\{s_{n} ; n=0,1, \ldots, N-1\right\}$, where $N=K M$, with $K$ denoting the spreading factor and $M$ denoting the number of data symbols for each user. The "overall" chip symbol, $s_{n}$, is given by

$$
s_{n}=\sum_{p=1}^{K_{U}} \xi_{p} s_{n, p}
$$

where

$$
s_{n, p}=c_{n, p} a_{\lfloor n / K\rfloor, p}
$$

is the $n$th chip for the $p$ th user $(\lfloor x\rfloor$ denotes 'larger integer not higher that $\left.x^{\prime}\right)$, with $\left\{a_{n, p} ; n=0,1, \ldots, M-1\right\}$ denoting the block of data symbols associated with the $p$ th user and $\left\{c_{n, p} ; n=0,1, \ldots, N-1\right\}$ denoting the corresponding spreading sequence (an orthogonal spreading is assumed). The power assigned to the $p$ th code is proportional to $\left|\xi_{p}\right|^{2}$.

To reduce the PMEPR of the transmitted signals, we consider the transmitter structure proposed in [8] and depicted in fig. 1A. The block of modified samples $\left\{s_{n}^{T x} ; n=\right.$ $0,1, \ldots, N-1\}$ is formed from the original block of samples $\left\{s_{n} ; n=0,1, \ldots, N-1\right\}$ in the following way: The original block of samples is passed to the frequency domain by a $N$ point DFT, leading to the block $\left\{S_{k} ; k=0,1, \ldots, N-1\right\}$. $N^{\prime}-N$ zeros are added to the block $\left\{S_{k} ; k=0,1 \ldots, N-1\right\}$ so as to form the augmented block $\left\{S_{k}^{\prime} ; k=0,1 \ldots, N^{\prime}-1\right\}$, with

$$
S_{k}^{\prime}= \begin{cases}S_{k}, & 0 \leq k<N / 2 \\ S_{k-N^{\prime}}, & N^{\prime}-N / 2 \leq k<N^{\prime} \\ 0, & \text { otherwise. }\end{cases}
$$

An IDFT brings the augmented block $\left\{S_{k}^{\prime} ; k=0,1 \ldots, N^{\prime}-\right.$ $1\}$ back to the time domain, resulting the block $\left\{s_{n}^{\prime} ; n=\right.$ $\left.0,1, \ldots, N^{\prime}-1\right\}$. These time-domain samples, which can be regarded as a sampled version of the DS-CDMA block, with the oversampling factor $M_{T x}=N^{\prime} / N,{ }^{2}$ are submitted to a nonlinear operation so as to reduce the corresponding PMEPR, leading to the modified samples

$$
s_{n}^{C}=g_{C}\left(\left|s_{n}^{\prime}\right|\right) \exp \left(j \arg \left(s_{n}^{\prime}\right)\right) .
$$

A possible nonlinear characteristic is

$$
g_{C}(R)= \begin{cases}R, & R<s_{M} \\ s_{M}, & R \geq s_{M}\end{cases}
$$

( $\left.R=\left|s_{n}^{\prime}\right|\right)$, which corresponds to an ideal envelope clipping, with clipping level $s_{M}$. A DFT brings the nonlinearly modified samples back to the frequency domain, leading to the block $\left\{S_{k}^{C} ; k=0,1, \ldots, N^{\prime}-1\right\}$, where a shaping operation is

\footnotetext{
${ }^{2}$ As shown in [8], $M_{T x}>1$ reduces the "in-band self-interference" effects of the nonlinearity, while increasing the "out-of-band self-interference" levels.
}

performed so as to obtain the block $\left\{S_{k}^{C F}=S_{k}^{C} G_{k} ; k=\right.$ $\left.0,1, \ldots, N^{\prime}-1\right\}$, with

$$
G_{k}= \begin{cases}1, & 0 \leq k<N / 2-1, N^{\prime}-N / 2 \leq k<N^{\prime} \\ 0, & \text { otherwise }\end{cases}
$$

(in a practical implementation, we simply remove the outof-band frequencies before the final IDFT). The rest of the transmitter is similar to a conventional, CP-assisted DS-CDMA transmitter (CP insertion, D/A conversion, etc.).

Whenever the number of users is high, conventional CDMA signals exhibit a Gaussian-like nature, with $E\left[s_{n}^{\prime}\right]=0$ and $E\left[\left|s_{n}^{\prime}\right|^{2}\right]=2 \sigma^{2}$. In that case, it can be shown that [8] the samples at the output of the nonlinear device can be decomposed into uncorrelated "useful" and "self-interference" components:

$$
s_{n}^{C}=\alpha s_{n}^{\prime}+d_{n},
$$

where $E\left[s_{n}^{\prime} d_{n^{\prime}}^{*}\right]=0$ and

$$
\begin{array}{r}
\alpha=\frac{E\left[R g_{C}(R)\right]}{E\left[R^{2}\right]}= \\
=\frac{1}{2 \sigma^{2}} \int_{0}^{+\infty} R g_{C}(R) \frac{R}{\sigma^{2}} \exp \left(-\frac{R^{2}}{2 \sigma^{2}}\right) d R .
\end{array}
$$

This means that

$$
S_{k}^{C F}=\alpha S_{k}^{\prime} G_{k}+D_{k} G_{k},
$$

where $E\left[D_{k}\right]=0$ and

$$
E\left[D_{k} D_{k^{\prime}}^{*}\right]= \begin{cases}N^{\prime} G_{d}(k), & k=k^{\prime} \\ 0, & k \neq k^{\prime},\end{cases}
$$

where $\left\{G_{d}(k) ; k=0,1, \ldots, N^{\prime}-1\right\}=\operatorname{DFT}\left\{R_{d}(n) ; n=\right.$ $\left.0,1, \ldots, N^{\prime}-1\right\}$, with

$$
R_{d}\left(n-n^{\prime}\right)=E\left[d_{n} d_{n^{\prime}}^{*}\right]
$$

denoting the autocorrelation of the self-interference component, which can be obtained analytically, as described in [8].

The samples $S_{k}^{T x}$ can also be decomposed into uncorrelated "useful" and "self-interference" terms:

$$
S_{k}^{T x}=\alpha S_{k}+D_{k}^{\prime \prime}, k=0,1, \ldots, N-1,
$$

where the block $\left\{D_{k}^{\prime \prime}, k=0,1, \ldots, N-1\right\}$ is obtained by removing the $N^{\prime}-N$ out-of-band samples of $\left\{D_{k}, k=\right.$ $\left.0,1, \ldots, N^{\prime}-1\right\}$. Therefore, the modified samples $s_{n}^{T x}$ can also be decomposed into uncorrelated "useful" and "selfinterference":

$$
s_{n}^{T x}=\alpha s_{n}+d_{n}^{\prime \prime},
$$

with $\left\{d_{n}^{\prime \prime}, n=0,1, \ldots, N-1\right\}=\operatorname{IDFT}\left\{D_{k}^{\prime \prime}, k=0,1, \ldots, N-\right.$ $1\}$. 

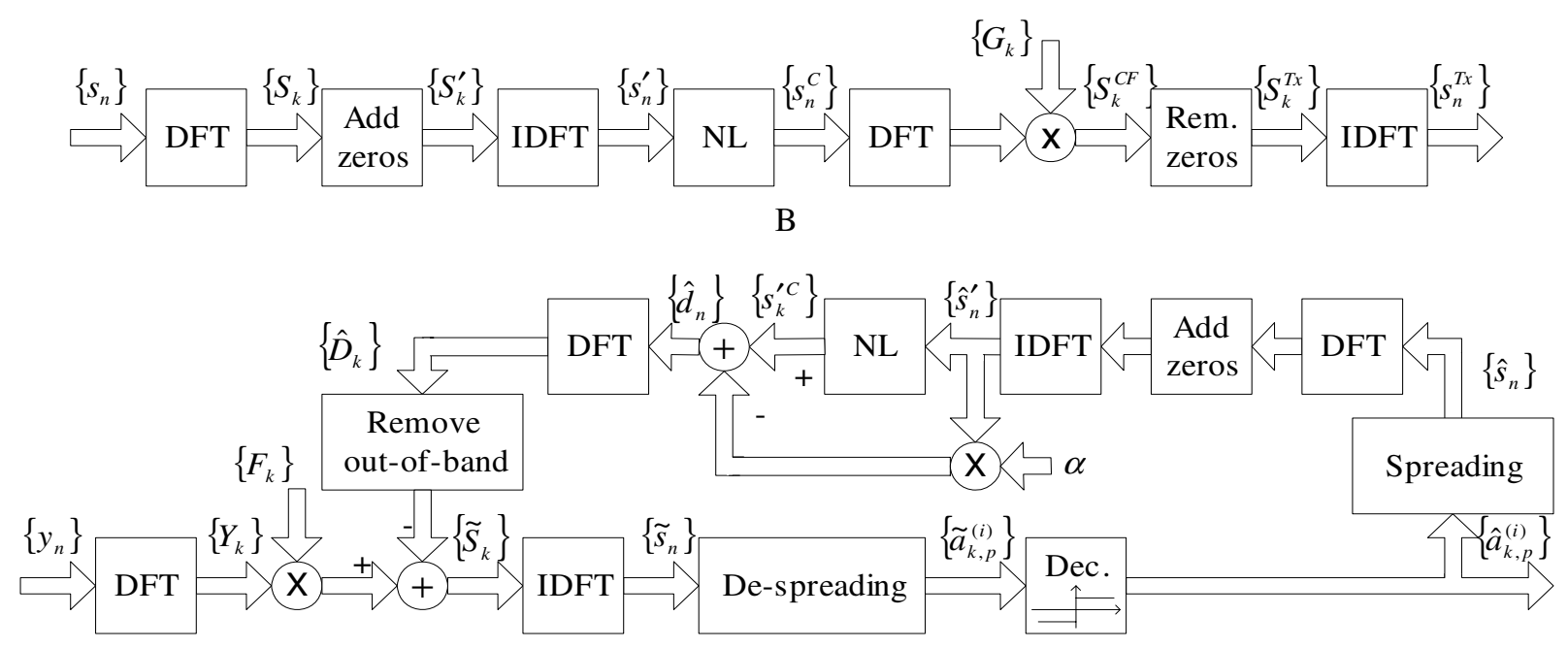

Fig. 1. Transmitter structure (A) and proposed receiver structure (B).

\section{Receiver Structure}

Since the orthogonality between users is lost in a timedispersive channel, we perform an FDE before the "despreading" procedure: after removing the $\mathrm{CP}$, the received timedomain block $\left\{y_{n} ; n=0,1, \ldots, N-1\right\}$ is passed to the frequency domain by a DFT leading to the block $\left\{Y_{k} ; k=\right.$ $0,1, \ldots, N-1\}=$ DFT $\left\{y_{n} ; n=0,1, \ldots, N-1\right\}$, where

$$
Y_{k}=H_{k} S_{k}^{T x}+N_{k}
$$

with $H_{k}$ and $N_{k}$ denoting the channel frequency response and the noise term for the $k$ th frequency (as usually, it is assumed that the $\mathrm{CP}$ is longer than the overall channel impulse response).

Since

$$
S_{k}^{T x}=\alpha S_{k}+D_{k}
$$

for the $N$ in-band frequencies, it can be shown that the optimum FDE coefficients in the MMSE sense (Minimum Mean Square Error) are given by

$$
F_{k}=\frac{\alpha^{*} E\left[\left|S_{k}\right|^{2}\right] H_{k}^{*}}{\left(E\left[\left|\alpha S_{k}\right|^{2} \mid\right]+E\left[\left|D_{k}\right|^{2}\right]\right)\left|H_{k}\right|^{2}+E\left[\left|N_{k}\right|^{2}\right]},
$$

where $E\left[\left|D_{k}\right|^{2}\right]$ can be analytically computed as described in [8]. The frequency-domain at the output of the FDE is then given by

$$
\tilde{S}_{k}=Y_{k} F_{k}
$$

The data block associated with the $p$ th user could be estimated by "de-spreading" the time-domain block at the FDE output, $\left\{\tilde{s}_{n} ; n=0,1, \ldots, N-1\right\}=\operatorname{IDFT}\left\{\tilde{S}_{k} ; k=\right.$ $0,1, \ldots, N-1\}$, i.e., from the samples

$$
\tilde{a}_{m, p}=\sum_{n=m K}^{m K+K} \tilde{s}_{n} c_{n, p}^{*} .
$$

It is shown in [8] that the equivalent "signal-to-selfinterference ratio" for the detection of the $p$ th spreading code is given by

$$
S I R_{p}^{e q}=\frac{K\left|\xi_{p}\right|^{2}}{K_{U} \overline{\left.\xi_{p}\right|^{2}}} S I R
$$

with

$$
\overline{\left|\xi_{p}\right|^{2}}=\frac{1}{K} \sum_{p^{\prime}}\left|\xi_{p^{\prime}}\right|^{2}
$$

and the signal-to-self-interference ratio on the transmitted signal given by

$$
S I R=\frac{\sum_{n} E\left[\left|\alpha s_{n}\right|^{2}\right]}{\sum_{n} E\left[\left|d_{n}^{\prime \prime}\right|^{2}\right]}=\frac{\sum_{k} E\left[\left|\alpha S_{k}\right|^{2}\right]}{\sum_{k} E\left[\left|D_{k}^{\prime \prime}\right|^{2}\right]} .
$$

Clearly, $S I R_{p}^{e q}$ decreases with $S I R$ and is smaller for codes with smaller attributed power (i.e., for codes where $\left|\xi_{p}\right|^{2}<$ $\overline{\left|\xi_{p}\right|^{2}}$ ).

It should be notted that the "overall interference" for detection purposes is approximately Gaussian-distributed when the spreading factor and the number of users is high [8]. This means that, if the data symbols are selected from a QPSK constellation under a Gray mapping rule the BER (Bit Error Rate) for an ideal Gaussian channel is approximately given by

$$
P_{b, p} \approx Q\left(\sqrt{S N R_{e q, p}}\right),
$$

where

$$
Q(x)=\int_{x}^{+\infty} \frac{1}{\sqrt{2 \pi}} \exp \left(-\frac{y^{2}}{2}\right) d y
$$

denotes the well-known Gaussian error function and $S N R_{e q, p}$ denotes an equivalent signal-to-noise ratio for the detection of the $p$ th code. This ratio can be obtained as described in [8].

Clearly, the nonlinear effects lead to some BER degradation relatively to conventional DS-CDMA schemes, especially when a low PMEPR is intended and/or for codes with small 
assigned power. This degradation results from both the "useless" power spent as a transmitted self-interference and the received self-interference being added to the channel noise. To improve the performance we consider the iterative receiver depicted in fig. 1B, where the self-interference components are estimated and removed, in an iterative way (it is assumed that the nonlinear characteristic adopted at the transmitter, $g_{C}(R)$, is known at the receiver).

After the de-spreading operation, the data estimates for each user $\hat{a}_{m, p}$ are obtained by submitting $\tilde{a}_{m, p}$ to an hard-decision device and used to form an estimate of the chip samples $\hat{s}_{n}$. By submitting these chip estimates to a replica of the nonlinear signal processing chain at the transmitter we can obtain an estimate of the self-interference component $\left\{\hat{D}_{k}\right\}$. For the next iteration, this self-interference estimate is removed form the frequency-domain samples at the output of the FDE. This procedure is repeated in an iterative way. For the first iteration $\hat{D}_{k}=0$.

It should be noted that, even if we were able to remove entirely the negative impact of the self-interference term in each frequency, we could not avoid a certain performance degradation due to the useless transmitted power inherent to the self-interference component. Therefore, for a QPSK constellation and an ideal Gaussian channel, the performance is lower-bounded by

$$
P_{b, L B}=Q\left(\sqrt{2 \eta_{S} \frac{E_{b}}{N_{0}}}\right)
$$

where

$$
\eta_{S}=\frac{\sum_{k} E\left[\left|\alpha S_{k}^{\prime}\right|\right]}{\sum_{k} E\left[\left|D_{k}^{\prime \prime}\right|\right]}
$$

is the performance degradation due to the useless power, which could be easily computed from the statistical characterization of the transmitted blocks of [8]. Fig. 2 shows the evolution of $\eta_{S}$ when $g_{C}(R)$ corresponds to an ideal envelope clipping, with normalized clipping level $s_{M} / \sigma$.

\section{Performance Results}

In this section we present a set of performance results concerning our improved receivers where an iterative cancelation of deliberate nonlinear distortion effects is carried out. The data symbols are selected from a QPSK constellation under a Gray mapping rule. We consider orthogonal spreading codes with length $N=K=K_{U}=256$ (i.e., a fully loaded system). $M_{T x}=N^{\prime} / N=2, g_{C}(R)$ corresponds to an ideal envelope clipping, with normalized clipping level $s_{M} / \sigma=1.0$, allowing PMEPR values of about $4.3 \mathrm{~dB}$ (for conventional DS-CDMA schemes with a large number of users $\mathrm{PMEPR} \approx 8.4$ ). The power amplifier at the transmitter is quasilinear within the range of variations of the input envelope. Perfect synchronization and channel estimation are assumed at the receiver.

Figs. 3 and 4 concern an ideal Gaussian channel. All users have the same assigned power in fig. $3\left(\xi_{p}=1\right)$; for fig.

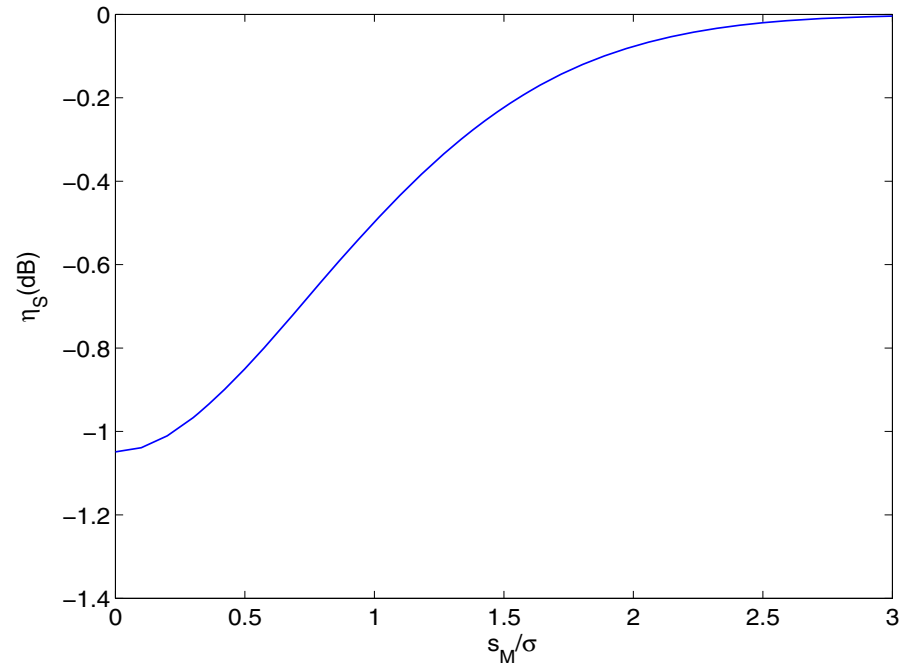

Fig. 2. Evolution of $\eta_{S}$ for an ideal envelope clipping, as a function of the normalized clipping level.

$4, \xi_{p}=1$ for $p=1,2, \ldots, K / 2$ and $\xi_{p}=2$ for the remaining users (i.e., the power assigned to half the users is $6 \mathrm{~dB}$ below the power assigned to the remaining users). Clearly the performance degradation due to the nonlinear distortion effects can be very high, especially for low-power users. By employing the proposed receiver with just two or three iterations, we can improve significantly the BER.

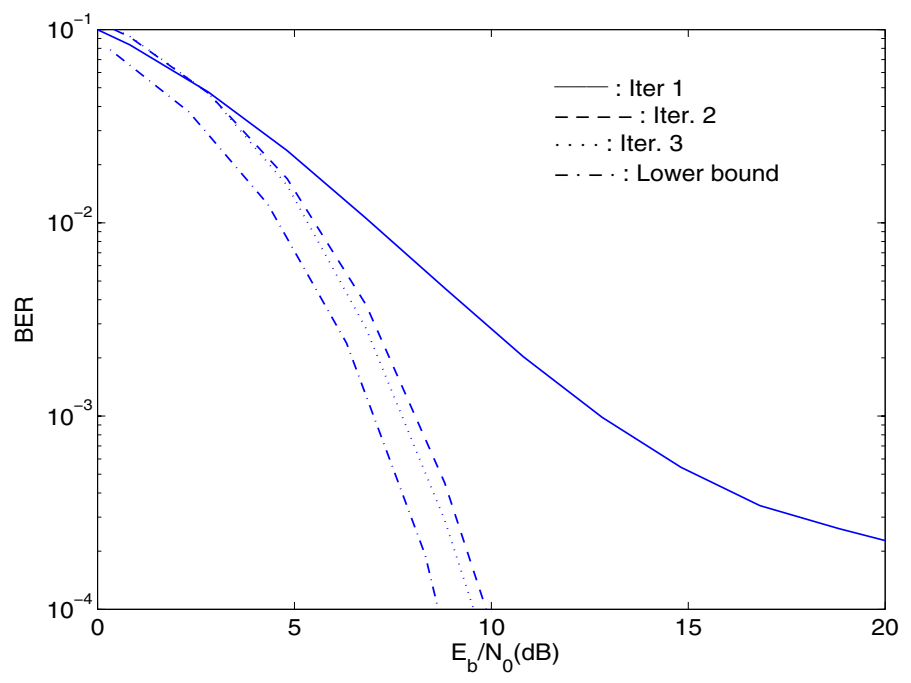

Fig. 3. BER performance for an ideal AWGN, when $\xi_{p}=1, p=$ $1,2, \ldots, K$.

For fig. 5, we have low-power and high-power users, as in fig. 4, but we considered a severely time-dispersive channel characterized by the power delay profile type $\mathrm{C}$ for the HIPERLAN/2 (HIgh PERformance Local Area Network) [11], with uncorrelated Rayleigh fading on the different paths. The duration of the useful part of the block is $5 \mu \mathrm{s}$ and 


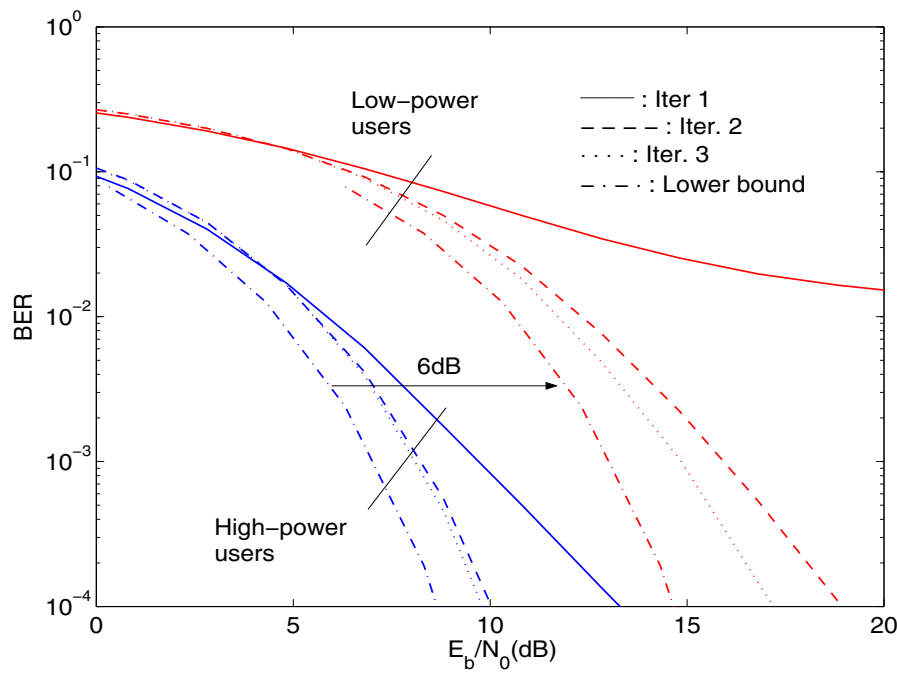

Fig. 4. BER performance, for an ideal AWGN, as a function of the $E_{b} / N_{0}$ of high-power users.

the $\mathrm{CP}$ has duration $1 \mu \mathrm{s}$ (similar results were obtained for other severely time-dispersive channels). Once again, we can improve significantly the BER with the proposed receiver, especially for low-power users.

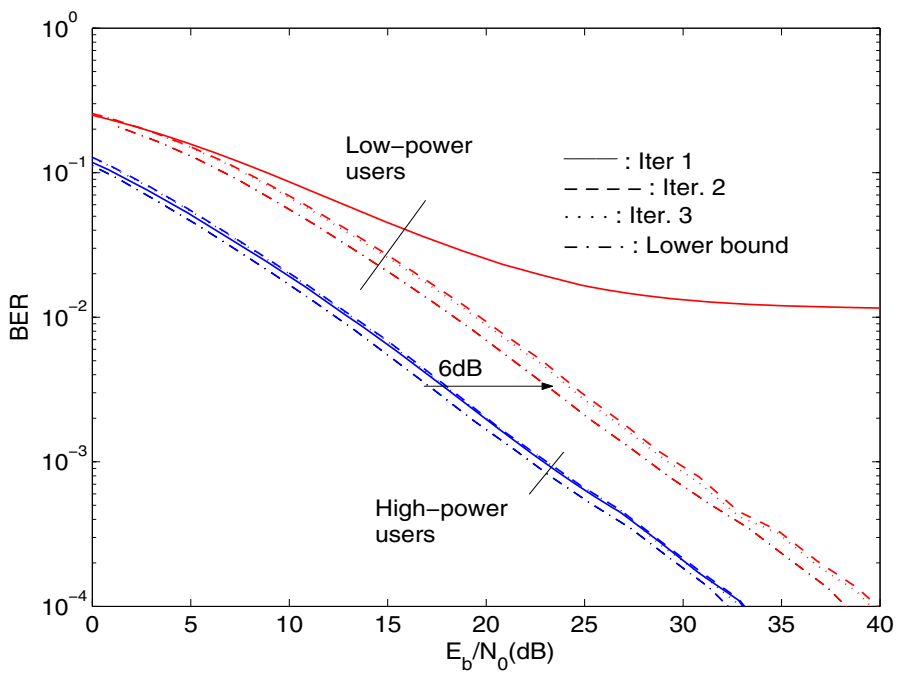

Fig. 5. As in fig. 4, but for a time-dispersive channel.

\section{Conclusions and Final Remarks}

In this paper we presented an improved receiver design where we perform an iterative estimation and cancelation of deliberate nonlinear distortion effects introduced at the transmitter. Our performance results show that we can improve significantly the performance with just a few iterations, even in the presence of strong nonlinear effects and/or for severely time-dispersive channels.

It should be noted that our receiver structure could easily be extended to the transmitter structures of [12] where the nonlinear and filtering operations are repeated, in an iterative way, so as to ensure a very low-PMEPR transmission. Is should also be noted that the data estimates employed in the feedback loop could be provided by a suitable channel decoder (as suggested in [13] for OFDM schemes), which is especially recommendable for scenarios where the nonlinear distortion effects are very sttrong (e.g., when the clipping level is very low and/or the nonlinear and filtering operations are repeated, in an iterative way (as in [12])). The performances on timedispersive channels could be further improved if the linear FDE is replaced by an IB-DFE (Iterative Block Decision Feedback Equalizer), as proposed in [14].

\section{References}

[1] A. Viterbi, CDMA: Principles of SS Communication, Addison Wesley, 1995.

[2] T. Ojamperä and R. Prasad, Wideband CDMA for Third Generation Mobile Communications, Artech House Publ., 1998.

[3] S. Barbarossa and F. Cerquetti, "Simple Space-Time Coded SSCDMA Systems Capable of Perfect MUI/ISI Elimination", IEEE Comm. Letters, Vol. 5, No. 12, pp. 471-473, Dec. 2001.

[4] K. Baum, T. Thomas, F. Vook, V. Nangia, "Cyclic-Prefix CDMA: An Improved Transmission Method for Broadband DS-CDMA Cellular Systems", IEEE WCNC, pp. 183-188, 2002.

[5] N.Guo and L.Milstein, Uplink Performance Evaluation of Multicode DS/CDMA Systems in the Presence of Nonlinear Distortions, IEEE J. on Sel. Areas in Comm., Vol. 18, No. 8, pp. 1418-1428, Aug. 2000.

[6] O.Väänänen, J.Vankka, T.Viero and K.Halonen, "Reducing the Crest Factor of a CDMA Downlink Signal by Adding Unused Channelization Codes", IEEE Comm. Letters, Vol. 6, No. 10, Oct. 2002.

[7] O.Väänänen, J.Vankka, T.Viero and K.Halonen, "Effect of Clipping in Wideband CDMA System and Simple Algorithm for Peak Windowing", World Wireless Congress, pp. 614-619. San Francisco, USA, May 28-31, 2002.

[8] R.Dinis and A.Palhau, "A Class of Signal Processing Schemes for Reducing the Envelope Fluctuations of CDMA Signals", IEEE Trans. on Comm., May 2005.

[9] J.Tellado, L.Hoo and J.Cioffi, "Maximum Likelihood Detection of Nonlinearly Distorted Multicarrier Symbols by Iterative Decoding", IEEE Trans. on Comm. Vol. 51, Feb. 2003.

[10] A.Gusmão and R.Dinis, "Iterative Receiver Techniques for Cancellation of Deliberate Nonlinear Distortion in OFDM-type Transmission", Int. OFDM Workshop'04, Dresden, Sep. 2004.

[11] ETSI, "Channel models for HIPERLAN/2 in Different Indoor Scenarios", ETSI EP BRAN 3ERI085B, pp. 1-8, March 1998.

[12] R.Dinis and A.Palhau,"A New Approach for Very Low-PMEPR Transmission in the Downlink of a DS/CDMA System", IEEE VTC'04(Spring), May 2004.

[13] A.Gusmão, P. Torres and R.Dinis, ”Low-PMEPR OFDM Transmission with an Iterative Receiver Technique for Cancellation of Nonlinear Distortion”, IEEE VTC'05(Fall), Dallas, Sep. 2005.

[14] P. Silva and R. Dinis, "An Iterative Frequency-Domain Decision Feedback Receiver for CDMA Systems”, , ISWCS'04, Sep. 2004. 